\title{
Profile of maraviroc: a CCR5 antagonist in the management of treatment-experienced HIV patients
}

This article was published in the following Dove Press journal:

HIVIAIDS - Research and Palliative Care

23 August 2010

Number of times this article has been viewed

\section{Thore Lorenzen \\ IFI Institute for Interdisciplinary Medicine, Hamburg, Germany}

Correspondence:Thore Lorenzen IFI Institute for Interdisciplinary Medicine, Lohmühlenstraße 5, 20099 Hamburg,

Germany

Tel +49-40-181885-3780

Fax +49-40-181885-3793

Email lorenzen@ifi-medizin.de

\begin{abstract}
Maraviroc is the first and, so far, the only licensed representative of the class of chemokine receptor type 5 (CCR5) inhibitors used for the treatment of human immunodeficiency virus (HIV) infection. Its safety and efficacy were demonstrated in several clinical trials, and its use was approved in 2007 by the responsible authorities. Some specific issues are correlated with maraviroc and its use. It is the only drug in the antiretroviral armamentarium, which does not interact with the viral enzymes but with a human receptor. Hence, it is able to be long-term effective only if the infecting virus uses, exclusively, the CCR5 receptor. Occurrence and detection of the CCR5 tropism are some of the great challenges of maraviroc use in treatment-experienced patients. Although up to $80 \%$ of naive patients harbor CCR5-tropic virus, the occurrence of CXCR4 or other tropisms increases with the duration of HIV infection and treatment. Nonetheless, maraviroc is a potent medication for eligible patients and helps to improve the outcome of antiretroviral treatment (ART) of HIV infection.
\end{abstract}

Keywords: CCR5 inhibitor, HIV infection, treatment-experienced patients, tropism

\section{Introduction}

Since the discovery of the human immunodeficiency virus-1 (HIV-1) in 1983 and the beginning of the battle against acquired immunodeficiency syndrome, the development of antiretroviral medication has succeeded substantially. The development and use of antiretroviral therapy (ARV) started in the mid to end of the 1980s with the first class of substances, the nucleoside reverse transcriptase inhibitors (NRTIs). ${ }^{1}$ In the following years, additional substances and classes have been introduced into clinical practice. Until now, 5 classes of ARV with more than 20 licensed drugs have been established for therapeutic use. The armamentarium predominantly includes substances influencing viral enzymes. Most of the proven substances inhibit the reverse transcriptase (RT) either as nucleoside antagonists or as non-NRTIs; others work as protease inhibitors (PIs) or integrase inhibitors by modifying the viral enzymatic activity. However, these substances are underlying a risk to select resistant viral mutants due to the variability of the virus under pharmacological pressure. The only antiretroviral class not interfering with the enzymes of the virus is the group of entry inhibitors.

Different substances, each with a specific mode of action, are represented within this class. The first one is enfuvirtide, which has been licensed in 2003. The potency of this fusion inhibitor is based on its ability to block the conformational change of gp41 and to avert the approach of virus and cell membrane. ${ }^{2}$ Despite the proven efficacy, enfuvirtide was not established as a routinely used drug because of its application mode 
as subcutaneous injections twice daily and the resultant side effects predominantly present as cutaneous irritations and painful indurations.

\section{Maraviroc}

Lacking the disadvantages of enfuvirtide, maraviroc was the first, and is still the only, approved entry inhibitor in oral formulation that showed potency and sustained efficacy to suppress HIV-1 viral load.

\section{Mechanism of action}

The mechanism of action of maraviroc is based on the need of HIV-1 to use a main receptor (CD4) and a coreceptor (CCR5 or CXCR4) on the surface of the target cells. HIV-1 attaches with its glycoprotein 120 (gp120) to the CD4 receptor. This is followed by a conformational change of gp120 and the coreceptor binding, which allows for the next step, the fusion of virus and cell membrane mediated by gp41.,4

In 1996, Huang et $\mathrm{al}^{5}$ demonstrated that a homozygous, 32-base pair mutation in the gene for CCR5 $(\Delta 32)$ protects against HIV infection by coding for a dysfunctional protein, which is not expressed on the cell surface. The later genetic studies demonstrated relatively high rates of $15 \%-20 \%$ of heterozygous $\Delta 32$ mutations in Caucasian populations, but rare cases in other populations. In 1996, Liu et $\mathrm{al}^{6}$ estimated that approximately $1 \%$ of the Caucasian population appeared to be homozygous for this $\Delta 32$ mutation. In fact, some of these individuals remained HIV-negative despite repeated exposure to HIV.

Based on this knowledge, it was found that maraviroc inhibits the attachment of HIV to its target cell via an allosteric modification of the CCR5 on the surface of the CD4 ${ }^{+}$ cells. It works as a small molecular CCR5 inhibitor through a binding in a cavity in the transmembrane CCR 5 receptor. This fixation of maraviroc changes the geometry of the transmembrane protein, which is originally needed for the binding of gp120 and CCR5. ${ }^{7-10}$

\section{Coreceptor usage of HIV}

Although HIV-1 predominantly uses the CCR 5 coreceptor for cell infection, it is not the only possibility to operate. Some viral strains have the ability to use a second coreceptor called CXCR4 to infect the target cells, and some viruses may use both CCR5 and CXCR4 receptors.

Epidemiological studies revealed that HIV-1 strains in ARV-naive patients predominantly exhibit CCR5 tropism (R5 viral variants). ${ }^{11-13}$ More than $80 \%$ of treatment-naive HIV-1-infected individuals carry R5 viruses, whereas approximately $20 \%$ show dual-tropic or mixed-tropic viruses (R5/X4 variants). Only a minority $(<1 \%)$ of viral strains are capable of using CXCR4 coreceptors exclusively (X4 variants). In treatment-experienced populations, $\mathrm{R} 5$ variants still account for $48 \%-62 \%$ of isolates and $\mathrm{R} 5 / \mathrm{X} 4$ variants are found in $34 \%-50 \%$ of these patients, whereas viruses that exclusively use the CXCR4 coreceptors for cell entry are seen in only $2 \%-4 \%$ of the pretreated population. ${ }^{14-16}$

The clinical relevance of CCR5 receptor variants, especially the homozygous one, is still unclear. Most recent studies suggest an association of $\Delta 32$ mutation and elevated mortality in cases of West Nile virus infections. ${ }^{17,18}$ In contrast, earlier studies suggested protection against chronic hepatitis B infection and rheumatoid arthritis or prolonged survival of renal transplant individuals. ${ }^{19-21}$ Additionally, an evaluation date back to the end of the last millennium discussed prevention of Yersinia infections (bubonic plague). ${ }^{22}$ In fact, many issues have been discussed but a final conclusion of the individual relevance of the CCR5 has not been established.

\section{Tropism testing in daily clinical practice}

As maraviroc cannot prevent cell infection in X4 or R5/X4 variants, it is necessary to detect viral tropism carefully in each individual before using it for ARV. As there are different methods to determine the tropism of the virus, it is still unclear which test is the most appropriate for routine clinical use. One can choose either phenotypic or genotypic methods, which differ substantially.

Phenotypic assays are based on the transfection of the virus into the cell culture, to mark the cell with a luciferase reporter gene, and on the determination of lyzed CCR5 or CXCR4 positive cell, in which a single viral cycle has been completed. Finally, the luciferase activity is measured by relative light units. ${ }^{23,24}$ Despite being considered to be the gold standard for the measurement of HIV-1 coreceptor usage, the phenotypic method has some disadvantages that should be kept in mind: the method is technically complex, expensive, and laborious. Only specialized laboratories with sophisticated equipments are able to operate these complicated methods. In fact, performance of a phenotypic tropism test takes up to 4 weeks from blood drawing and costs between $\$ 750$ and $\$ 1000$.

In contrast, genotypic assays may be performed within some days, and the cost is restricted to the performance of a gene amplification, mostly of the V3 region. Subsequently, the amplification products are analyzed in a sequencer, and the generated sequences are correlated with 
several standard sequences. For interpretation, some of the algorithm systems predicting coreceptor usage are available online. These systems, such as Webcat, WebPSSM, and geno2pheno[coreceptor], are accessible via the Internet. Established laboratories use these systems to determine the coreceptor usage. A restriction of these systems is the relatively low sensitivity and specificity, but combining 2 or 3 systems enhances the recall ratio. ${ }^{25-27}$

\section{Clinical development of maraviroc}

Because of the importance of coreceptors for viral entry and the knowledge of research in other entry inhibitors, the Pfizer Global Research and Development discovered maraviroc (UK-427,857) as a highly promising substance to block the CCR5 receptor effectively.

Clinical studies started with dose-finding and safety issues as short-term monotherapy administration in treatment-naive patients and in antiretroviral treatment (ART)-experienced patients, who had to be on treatment for at least 8 weeks (A4001007 and A4001015). Participation was restricted to individuals with confirmed R5 viral variants, viral load $>5,000$ HIV-1 copies/mL, and moderate immunodeficiency with still more than $250 \mathrm{CD}^{+}$cells $/ \mathrm{mm}^{3}$. Results of these trials demonstrated efficacy of maraviroc in both naive and experienced patients with R5 but not with $\mathrm{X} 4$ viruses. ${ }^{28}$

Following trials (MOTIVATE-1 and MOTIVATE-2) evaluated the efficacy of maraviroc in treatment-experienced patients harboring R5-tropic variants. Both trials were double-blind, placebo-controlled, multicenter phase $2 \mathrm{~b} / 3$ studies, investigating maraviroc plus optimized background therapy (OBT) vs placebo plus OBT in viremic patients (viral load $>5000$ copies/mL) carrying CCR5-tropic virus. The treatment groups were also divided into patients receiving maraviroc once or twice daily. MOTIVATE-1 was conducted in the United States and Canada, whereas the identically designed MOTIVATE-2 trial enrolled patients in Europe, Australia, and North America. The primary end point of both studies was viral load change in HIV-1 RNA from baseline to week 48. According to the inclusion criteria, all patients had to be triple-class experienced. The results showed a significant advantage for patients in the maraviroc groups: viral load declined by $1.66 \log _{10}$ copies/mL and $1.82 \log _{10}$ copies/ $\mathrm{mL}$ for once-daily and twice-daily administration of drug, respectively, whereas in the placebo arm, it declined only by $0.80 \log _{10}$ copies/mL in MOTIVATE-1. Nearly the same results could be found in MOTIVATE-2: 1.72 and $1.87 \log _{10}$ reduction vs $0.76 \log _{10}$ reduction, respectively. The study data also showed a superior response of CD4 cells in the treatment arms: the mean increase of CD4 cells in MOTIVATE-1 was 113 and 122 cells/ $\mu \mathrm{L}$ for once-daily and twice-daily administration, respectively, vs 54 cells $/ \mu \mathrm{L}$ in the placebo arm. In MOTIVATE-2, the CD4 cells increased by 122 and 128 cells/ $\mu \mathrm{L}$ for once-daily and twice-daily administration, respectively, (verum) and 69 cells $/ \mu \mathrm{L}$ (placebo).

The pooled safety analysis of both studies demonstrated no statistical significant differences in treatment-related adverse events, which indicates a good compatibility of maraviroc. ${ }^{29-31}$

The results of the MOTIVATE studies led to the approval of maraviroc, with the restriction for use in pretreated patients harboring only R5-tropic viral strains.

A further study called MERIT compared maraviroc plus zidovudine/lamivudine with efavirenz plus the same backbone in ARV-naive patients. The original study missed its goal to demonstrate noninferiority of maraviroc correlated to efavirenz according to the rate of viral load $<50$ copies/ $\mu \mathrm{L}$ after 48 weeks. However, it demonstrated the very good compatibility of the CCR5-inhibitor: patients following the efavirenz-containing regimen discontinued their therapy thrice, more often than maraviroc recipients did.

Later analyses verified that the original goal of noninferiority was missed because of the lacking sensitivity of the primarily used test to detect viral tropism. A retrospective analysis, with an exclusion of the additionally found $\mathrm{X} 4$-tropic virus in patients, by using an advanced version of the tropism test demonstrated the expected noninferiority of maraviroc. Additionally, patients in the maraviroc arm demonstrated a significantly higher CD4 cell increase compared with the efavirenz arm.

Nonetheless, one of the maraviroc arms was conducted as once-daily administration of study drug. This arm was discontinued prematurely due to a lack of efficacy. ${ }^{32}$ A compilation of maraviroc-associated data of the MOTIVATE and MERIT trials is shown in Table 1.

\section{Resistance issues of maraviroc}

One of the most problematic issues in battling HIV is the development of resistances to antiretroviral drugs. Regularly, selection of resistant mutants develops under pharmaceutical pressure, while viral replication occurs due to the irregular intake of medication and/or suboptimal drug levels. In the RT, the protease, and the integrase, occurrence of mutations may be strongly associated with the drug used: eg, lamivudine regularly causes a M184 V mutation in the RT gene locus, which is associated with a resistance to lamivudine but reduces viral fitness significantly. ${ }^{33}$ On the other hand, 
Table I 96-week results of maraviroc studies in patients harboring CCR5-tropic virus

\begin{tabular}{|c|c|c|c|}
\hline Study & $\begin{array}{l}\text { MOTIVATE I and } 2 \\
\text { pooled data }\end{array}$ & $\begin{array}{l}\text { MERIT primary } \\
\text { analysis }\end{array}$ & $\begin{array}{l}\text { MERIT post } \\
\text { hoc analysis }\end{array}$ \\
\hline No of patients who used maraviroc twice a day & 426 & 360 & 311 \\
\hline \multicolumn{4}{|l|}{ Baseline characteristics } \\
\hline HIV-I RNA level, mean, copies/mL & $4.85 \log _{10}$ & $4.86 \log _{10}$ & $4.88 \log _{10}$ \\
\hline CD4 cell count, median & 167 cells $/ \mu \mathrm{L}$ & $24 \mathrm{I}$ cells/ $/ \mu \mathrm{L}$ & 236 cells $/ \mu \mathrm{L}$ \\
\hline \multicolumn{4}{|l|}{ Virological and immunological responses } \\
\hline HIV-I RNA: change from & -1.84 & Data on file & Data on file \\
\hline \multicolumn{4}{|l|}{ baseline, mean, $\log _{10}$ copies $/ \mathrm{mL}$} \\
\hline HIV-I RNA: $<400$ copies/mL & 50 & 61.7 & 64.3 \\
\hline HIV-I RNA: $<50$ copies $/ \mathrm{mL}$ & 40 & 57.8 & 60.5 \\
\hline CD4 cell count, change from baseline, mean, cells $/ \mathrm{mm}^{3}$ & 187 & 224 & 224 \\
\hline Virological failure after $96 \mathrm{wk} \%$ & 25 & 22 & 22 \\
\hline
\end{tabular}

Abbreviations: CCR5, C-C chemokine receptor type 5; HIV, human immunodeficiency virus.

some protease-associated mutations, such as L90M, may be initiated by 1 drug (eg, saquinavir), but may have impact on other drugs of the same class (eg, atazanavir and nelfinavir). ${ }^{34}$ These mechanisms of direct or cross-resistance are characteristic for drugs interfering with viral enzymes.

In CCR5 inhibitors, there are more escape mechanisms for the virus; primarily, the use of the above-mentioned coreceptor may change: a R5-using virus might be successfully defeated but the virus may switch to use CXCR4 as coreceptor. In an in vitro study recently conducted, it has been shown that the presence of maraviroc in a cell culture does not lead to a switch of R5 viral strains to X4 variants. Thus, it appears not to be common for a virus to switch from its origin to another tropism, it seems to be rather unusual. Therefore, another possibility appears to be more reliable with respect to the development of resistance: a preexisting $\mathrm{X} 4$ virus minority may get selected due to the R5 suppression, and as result may lead to resistance against CCR5 inhibitors. However, there are also other mechanisms forcing resistance to maraviroc: as maraviroc directly interacts with the human CCR5, the viral correlate, the env gene with its gp120 and gp41, may mutate. Studies showed the changes of the V3 loop of gp120 lead to resistance, but mutations have also been reported in other regions of gp120 and gp41 due to the enormous variability of the env gene. Additionally, the development of resistance in the presence of CCR 5 inhibitors does not select always the same mutations. ${ }^{35-39}$ Consequently, the prediction of resistance in CCR5 inhibitors may be more complicated and more difficult to interpret than it is with the RT or PIs.

A third possibility for the virus to attain infectiveness of its combatant maraviroc or other CCR5 inhibitors is the ability to bind to the coreceptor despite specific drug is bound, whether through a competitive replacement due to higher affinity than the drug or because of the possibility to bind despite a drug-bound receptor. ${ }^{40,41}$

\section{When to use maraviroc}

Taking the above-mentioned facts into account, the consequences of therapeutic use still remain elusive. Nearly, $80 \%$ of all antiretroviral naive patients harbor R5-tropic virus, and therefore, would be eligible for using this drug. However, until 2009, the worldwide approvals for maraviroc indicate its use only for pretreated patients with R5-tropic HIV-1, partly with this requirement alone or partly with the necessity of resistances to other antiretroviral drug. Since 2009, maraviroc is licensed in the United States under a statement, "for combination antiretroviral treatment of adults infected with only CCR5-tropic HIV-1" (US Food and Drug Administration approval November 20, 2009), which means that it can be used also in naive patients. However, this statement is directly followed by a special note: “... more subjects treated with SELZENTRY ${ }^{\circledR}$ experienced virologic failure and developed lamivudine resistance compared to efavirenz ...," which puts the indication into perspective. Hence, the use of maraviroc in treatment-naive individuals would be according to the applicable label, but may induce a higher risk for the patients to develop virological failure and acquiring resistances. Therefore, maraviroc should be the first choice in treatment-naive patients only under special conditions and thorough surveillance.

In treatment-experienced subjects, the MOTIVATE studies demonstrated a benefit of maraviroc compared with the placebo arms in virological and immunological responses. However, 955 individuals (29.4\%) of 3,244 screened patients were directly excluded from the studies due to the occurrence of X4 or dual $/$ mixed tropism. Overall, in only $61 \%$ of the initially tested patients, an R5 tropism could be clearly 
demonstrated. ${ }^{29}$ These results were generated with a later revised phenotypic assay (Trofile ${ }^{\mathrm{TM}}$, Monogram Bioscience, South San Fransico, California, USA). Unfortunately, the possible subsequently generated data obtained using the enhanced Trofile assay, which is more sensitive, are not applicable. Nonetheless, the virological activity of maraviroc and the good compatibility could be demonstrated in the studies and in clinical practice. However, before starting $\mathrm{ARV}$, it needs to be demonstrated that the patient is harboring R5-tropic virus, exclusively. Hence, maraviroc serves as an enrichment of the armamentarium for HIV treatment.

\section{Disclosure}

The author discloses no conflicts of interest.

\section{References}

1. Nakashima H, Matsui $T$, Harada $S$, et al. Inhibition of replication and cytopathic effect of human T cell lymphotropic virus type III/ lymphadenopathy-associated virus by 3'-azido-3'-deoxythymidine in vitro. Antimicrob Agents Chemother. 1986;30(6):933-937.

2. Cervia JS, Smith MA. Enfuvirtide (T-20): a novel human immunodeficiency virus type 1 fusion inhibitor. Clin Infect Dis. 2003;37(8): 1102-1106.

3. Dragic T. An overview of the determinants of CCR 5 and CXCR4 co-receptor function. J Gen Virol. 2001;82(Pt 8):1807-1814.

4. Alkhatib G. The biology of CCR5 and CXCR4. Curr Opin HIV AIDS. 2009;4(2):96-103.

5. Huang Y, Paxton WA, Wolinsky SM, et al. The role of a mutant CCR5 allele in HIV-1 transmission and disease progression. Nat Med. 1996;2:1240-1243.

6. Liu R, Paxton WA, Choe S, et al. Homozygous defect in HIV-1 coreceptor accounts for resistance of some multiply-exposed individuals to HIV-1 infection. Cell. 1996;86:367-377.

7. Dragic T, Trkola A, Thompson DA, et al. A binding pocket for a small molecule inhibitor of HIV-1 entry within the transmembrane helices of CCR5. Proc Natl Acad Sci U S A. 2000;97:5639-5644.

8. Tsamis F, Gavrilov S, Kajumo F, et al. Analysis of the mechanism by which the small-molecule CCR5 antagonists $\mathrm{SCH}-351125$ and SCH350581 inhibit human immunodeficiency virus type 1 entry. $J$ Virol. 2003;77:5201-5208.

9. Watson C, Jenkinson S, Kazmierski W, et al. The CCR5 receptor-based mechanism of action of 873140 , a potent allosteric noncompetitive HIV entry inhibitor. Mol Pharmacol. 2005;67:1268-1282.

10. Seibert C, Ying W, Gavrilov S, et al. Interaction of small molecule inhibitors of HIV-1 entry with CCR5. Virology. 2006;349: $41-54$.

11. Brumme ZL, Goodrich J, Mayer HB, et al. Molecular and clinical epidemiology of CXCR4-using HIV-1 in a large population of antiretroviralnaive individuals. J Infect Dis. 2005;192(3):466-474.

12. Moyle GJ, Wildfire A, Mandalia S, et al. Epidemiology and predictive factors for chemokine receptor use in HIV-1 infection. J Infect Dis. 2005;191(6):866-872.

13. Demarest J, Sparks S, Watson C, McDanal C, Kenakin TP, Shibayama S. Prolonged and unique binding of a novel CCR antagonist, 873140. [Abstract H-211]. Paper presented at: 44th ICAAC; 2004; Washington.

14. Whitcomb JM, Huang W, Fransen S, et al. Analysis of baseline enfuvirtide (T20) susceptibility and co-receptor tropism in two-phase III study populations. [Abstract 557]. Paper presented at: 10th Conference on Retroviruses and Opportunistic Infections; 2003; Boston, MA.
15. Paxinos EE, Fransen S, Huang W, Wrin T, Whitcomb JM. Measuring HIV-1 co-receptor tropism using a recombinant virus assay. [Abstract H-2040]. Paper presented at: 42nd ICAAC; 2002; San Diego, CA.

16. Wilkin T, Su Z, Kuritzkes D, et al. Co-receptor tropism in patients screening for ACTG 5211, a phase 2 study of vicriviroc, a CCR5 inhibitor. [Abstract 655]. Paper presented at: 13th Conference on Retroviruses and Opportunistic Infections; 2006; Denver, CO.

17. Glass WG, Lim JK, Cholera R, et al. Chemokine receptor CCR5 promotes leukocyte trafficking to the brain and survival in West Nile virus infection. $J$ Exp Med. 2005;202:1087-1098.

18. Glass WG, McDermott DH, Lim JK, et al. CCR5 deficiency increases risk of symptomatic West Nile virus infection. $J$ Exp Med. 2006;203:35-40.

19. Fischereder M, Luckow B, Hocher B, et al. CC chemokine receptor 5 and renal-tranplant survival. Lancet. 2001;357:1758-1761.

20. Prahalad S. Negative association between the chemokine receptor CCR5-[Delta]32 polymorphism and rheumatoid arthritis: a metaanalysis. Genes Immun. 2006;7:264-268.

21. Thio CL, Astemborski J, Bashirova A, et al. Genetic protection against hepatitis B virus conferred by CCR5Delta32: evidence that CCR5 contributes to viral persistence. J Virol. 2007;81: 441-445.

22. Stephens JC, Reich DE, Goldstein DB, et al. Dating the origin of the CCR5-Delta32 AIDS-resistance allele by the coalescence of haplotypes. Am J Hum Genet. 1998;62:1507-1515.

23. Braun P, Wiesmann F. Phenotypic assays for the determination of coreceptor tropism in HIV-1 infected individuals. Eur J Med Res. 2007;12(9):463-472.

24. Low AJ, Swenson LC, Harrigan PR. HIV coreceptor phenotyping in the clinical setting. AIDS Rev. 2008;10(3):143-151.

25. Sierra S, Kaiser R, Thielen A, et al. Genotypic coreceptor analysis. Eur J Med Res. 2007;12:453-462.

26. Raymond S, Delobel P, Mavigner M, et al. Correlation between genotypic predictions based on $\mathrm{V} 3$ sequences and phenotypic determination of HIV-1 tropism. AIDS. 2008;22(14):F11-F16.

27. Lin NH, Kuritzkes DR. Tropism testing in the clinical management of HIV-1 infection. Curr Opin HIV AIDS. 2009;4(6):481-487.

28. Fätkenheuer G, Pozniak AL, Johnson MA, et al. Efficacy of short-term monotherapy with maraviroc, a new CCR5 antagonist, in patients infected with HIV-1. Nat Med. 2005;11:1170-1172.

29. Gulick RM, Lalezari J, Goodrich J, et al. Maraviroc for previously treated patients with R5 HIV-1 infection. $N$ Engl $J$ Med. 2008;359(14):1429-1441.

30. Fatkenheuer G, Nelson M, Lazzarin A, et al. Subgroup analyses of maraviroc in previously treated R5 HIV-1 infection. $N$ Engl $J$ Med. 2008;359:1442-1455.

31. Asmuth DM, Goodrich J, Cooper DA, et al. CD4 ${ }^{+}$T-cell restoration after 48 weeks in the Maraviroc Treatment-Experienced Trials MOTIVATE 1 and 2. J Acquir Immune Defic Syndr. [Epub 2009 ahead of print].

32. Cooper DA, Heera J, Goodrich J, et al. Maraviroc versus efavirenz, both in combination with zidovudine-lamivudine, for the treatment of antiretroviral-naive subjects with CCR5-tropic HIV-1 infection. J Infect Dis. 2010;201(6):803-813.

33. Miller V, Stark T, Loeliger AE, Lange JM. The impact of the M184V substitution in HIV-1 reverse transcriptase on treatment response. HIV Med. 2002;3(2):135-145.

34. Cheung PK, Wynhoven B, Harrigan PR. 2004: which HIV-1 drug resistance mutations are common in clinical practice? AIDS Rev. 2004;6(2):107-116.

35. Trkola A, Kuhmann SE, Strizki JM, et al. HIV-1 escape from a small molecule, CCR5-specific entry inhibitor does not involve CXCR4 use. Proc Natl Acad Sci U SA. 2002;99:395-400.

36. Marozsan AJ, Kuhmann SE, Morgan T, et al. Generation and properties of a human immunodeficiency virus type 1 isolate resistant to the small molecule CCR5 inhibitor, SCH-417690 (SCH-D). Virology. 2005;338:182-199. 
37. Baba M, Miyake H, Wang X, et al. Isolation and characterization of human immunodeficiency virus type 1 resistant to the smallmolecule CCR5 antagonist TAK-652. Antimicrob Agents Chemother. 2007;51:707-715.

38. Westby M, Smith-Burchnell C, Mori J, et al. Reduced maximal inhibition in phenotypic susceptibility assays indicates that viral strains resistant to the CCR5 antagonist maraviroc utilize inhibitor-bound receptor for entry. J Virol. 2007;81:2359-2371.

39. Ogert RA, Wojcik L, Buontempo C, et al. Mapping resistance to the CCR5 co-receptor antagonist vicriviroc using heterologous chimeric HIV-1 envelope genes reveals key determinants in the C2-V5 domain of gp120. Virology. 2008;373:387-399.
40. Nolan KM, Del Prete GQ, Jordan AP, et al. Characterization of a human immunodeficiency virus type $1 \mathrm{~V} 3$ deletion mutation that confers resistance to CCR5 inhibitors and the ability to use aplaviroc-bound receptor. J Virol. 2009;83(8):3798-3809.

41. Tilton JC, Amrine-Madsen H, Miamidian JL, et al. HIV type 1 from a patient with baseline resistance to CCR5 antagonists uses drug-bound receptor for entry. AIDS Res Hum Retroviruses. 2010;26(1):13-24.

\section{Publish your work in this journal}

HIV/AIDS - Research and Palliative Care is an international, peerreviewed open-access journal focusing on advances in research in HIV, its clinical progression and management options including antiviral treatment, palliative care and public healthcare policies to control viral spread. The journal welcomes original research, basic science, clinical \& epidemiological studies, reviews \& evaluations, expert opinion \& commentary, case reports \& extended reports. The manuscript management system is completely online and includes a very quick and fair peer-review system. Visit http://www.dovepress.com/ testimonials.php to read real quotes from published authors.

Submit your manuscript here: http://www.dovepress.com/hivaids---research-and-palliative-care-journal 